\title{
Evaluación neuropsicológica de una muestra de niños de 5 a 12 años con instrucción escolar bilingüe
}

\author{
Javier Galarza del Angel \\ Alfredo Padilla López \\ Julieta Bonilla \\ Universidad Autónoma de Baja California, México
}

\begin{abstract}
RESUMEN
Como parte de un proyecto más amplio, en el que se pretende obtener datos normativos de diferentes muestras de niños con diferentes tipos de instrucción y de diferente nivel socioeconómico, se evaluaron 176 niños de ambos sexos con edades de entre cinco y doce años y del nivel socioeconómico medio. Los niños recibían instrucción escolar bilingüe. Se formaron cuatro grupos de edad (5-6, 7-8, 9-10 y 11-12) y se dividieron por sexo. Se les aplicaron las siguientes pruebas: Prueba de Denominación de Boston, Prueba de Memoria Verbal Secuencial, Prueba de Fichas, Prueba de Figuras Superpuestas, Prueba de Fluidez Verbal (FAS) en las categorías fonológica y semántica. También se hizo una revisión ortográfica de la ejecución de los niños en la categoría fonológica. Se encontraron diferencias entre los grupos de edad en todas las pruebas, pero no se encontraron diferencias entre los sexos en ninguna de las pruebas. Se comparan los resultados con los reportados por Ardila y Rosselli (1994). Se continuarán las siguientes fases del proyecto en las que se evaluarán niños de diferente nivel socioeconómico y con instrucción escolar monolingüe.
\end{abstract}

Palabras-clave: evaluación neuropsicológica; neuropsicología infantil; datos normativos.

\section{ABSTRACT}

\section{Neuropsychological assessement of 5 to 12 year-old children with bilingual schoolar education}

As a part of a wider study, 176 children were evaluated through a neuropsychological battery. Children were 5 to 12 year-old (both sex), they were in medium socio-economic level and they were in a bilingual school. Children were divided by sex, and by age group (5-6, 7-8, 9-10 and 11-12 yearold). Neuropsychological battery included the Boston Naming Test, Token Test, Superimposed Figures (Poppelreuter type), a Verbal Fluency Test (FAS Test) and a Sequential Verbal Memory Test. No differences were found between sex in any of the applied tests. There was a significative difference among age groups for all the tests that were applied. Results are compared with some other reported (Ardila \& Rosselli, 1994). This study will continue in evaluation of another socioeconomic levels and scholar education.

Keywords: neuropsychological assessment; children neuropsychology; normative data.

El propósito de este trabajo es obtener parámetros de ejecución en niños de cinco a doce años de edad en la aplicación de una batería de pruebas neuropsicológicas.

Actualmente se utiliza una gran cantidad de pruebas para evaluar diferentes procesos neuropsicológicos (Lezak, 1983; Spreen y Strauss, 1991). Sin embargo las pruebas neuropsicológicas pueden ser sensibles a los factores socio-culturales (Ardila, 1995), y en este sentido se puede esperar que la gente de Perú, por ejemplo, obtenga diferentes puntajes que la de México o de Italia. Más aún, la gente que vive en diferentes regiones de un mismo país podría obtener diferentes puntajes en ciertas pruebas.

En México han habido estudios para estandarizar algunas pruebas neuropsicológicas (Galindo, Cortés, Salvador, Chatelain y San-Esteban, 1992) y se han propuesto baterías neuropsicológicas (Ardila y
Ostrosky, 1991; Canseco, 1988). Evidentemente estos esfuerzos no han sido suficientes para desarrollar datos normativos para la diversidad cultural mexicana y por lo tanto el hecho es que el neuropsicólogo no tiene suficientes y confiables datos normativos. Se han reportado algunas investigaciones para obtener datos sobre procesos muy específicos. Por ejemplo, Curro (1993) presentó una propuesta para evaluar memoria auditiva, visual y táctil en niños. Por otra parte, Valdez, Ramírez y Nava (1993) desarrollaron una prueba para evaluar memoria de figuras y palabras.

En Colombia Ardila y Rosselli (1994) aplicaron una batería neuropsicológica a niños de cinco a doce años de edad y obtuvieron datos normativos de la ejecución de los niños. También existen algunas propuestas para evaluación de niños: Lopera (1992) propone un método para evaluar niños desde la perspectiva de la neurología, en tanto que Ferre 
(1992) propone otro para evaluar desde el punto de vista de la neuropsicología.

El presente trabajo tuvo como objetivos:

1. Obtener parámetros de ejecución en niños normales de cinco a doce años de edad.

2. Evaluar si existen diferencias de ejecución entre los diferentes grupos de edad.

3. Evaluar si existen diferencias de ejecución entre los sexos.

\section{MÉTODO}

\section{Sujetos}

Se evaluaron 176 niños de ambos sexos con edades entre los cinco y doce años. Los niños fueron seleccionados en forma no probabilística debido a problemas para conseguir permiso entre las escuelas locales. Los niños cursaban desde el tercer grado de pre-escolar hasta el primer año de secundaria y estaban aparentemente sanos; ninguno había presentado enfermedades serias, ni tampoco alteraciones de tipo neurológico. El colegio al que asistían es bilingüe, dado que este trabajo forma parte de un proyecto más amplio en que se contempla la evaluación de niños de diferentes estratos socioeconómicos y de diferentes condiciones de instrucción escolar (bilingüe y monolingüe). Mexicali, es una ciudad fronteriza del norte de México. Se formaron 8 grupos divididos por sexo (niños-niñas) y por edad (5-6, 7-8, 9-10 y 11-12 años). En cada grupo había un promedio de 20 niños.

\section{Materiales}

Se aplicaron los siguientes pruebas:

1. Prueba de Denominación de Boston (PB). Se utilizó la versión española (Goodglass y Kaplan 1972/1986).

2. Prueba de Fichas (PF). Versión abreviada (De Renzi y Faglioni, 1978).

3. Prueba de Fluidez Verbal. Fonológica (FASF) (frutas y animales) Semántica (FASS) (se utilizaron las letras F, A, y S). Además se hizo una revisión ortográfica (FASO) en la que se revisaba si los niños además de identificar el sonido con el que iniciaba la palabra podía mantenerse en la regla ortográfica, es decir que si se le pedía que dijera palabras que iniciaran con S, efectivamente mencionara palabras como silla o sol; pero no zurdo, cerillo o cielo.

4. Prueba de Memoria Verbal Secuencial (PMVS) (Ardila y Rosselli, 1994). Esta prueba evalúa la habilidad del individuo para memorizar en forma secuencial y ordenada una serie de 10 palabras. En este trabajo solo se aplicó en la condición de recuerdo inmediato.

5. Figuras Superpuestas (FS) Se le pedía a los niños que reconocieran figuras dibujadas en forma superpuestas en cuatro tarjetas (2, 3, 4 y 5 figuras en cada tarjeta).

6. Escala de Memoria de Wechsler. Se utilizó la versión usada por Ardila y Rosselli (1994). Se repitió la aplicación de las subescalas 5, 6 y 7 en forma demorada inmediatamente despues de presentar la subescala 7.

7. Figura Compleja de Rey-Osterrieth (FCRO). Se evaluó la copia y la reproducción inmediata de memoria.

En este trabajo se reportan únicamente $\mathrm{PB}, \mathrm{PF}$, FASF, FASO, FASS, PMVS, y FS.

\section{Procedimiento}

Los niños fueron evaluados en forma individual en sesiones que duraban entre $1 \mathrm{hr}$. 15 mins. para los niños mayores, y 1 hr. 45 mins. para los menores. Se visitó cada uno de los salones de clase y se les explicaba a los niños qué se iba a hacer. Previamente se había realizado un sorteo y se les notificaba a los niños quiénes habían sido seleccionados y se les decía que ellos podían elegir no participar en el estudio. Todos los niños aceptaron. No se les recompensaba de ninguna forma.

\section{RESULTADOS}

En la PB se observa que los niños tienen en promedio puntajes más bajos que las niñas; esta diferencia, sin embargo, no es significativa. Al comparar los puntajes obtenidos en la PB por los niños y niñas en los diferentes grupos de edad se encontraron diferencias entre las medias: F (3, 172)=97.709, p<0.000. Las niñas de 5-6 años obtuvieron 25.05 (d.e.=6.41), en tanto que las niñas de 11-12 obtuvieron 42.91 (d.e.=4.16). El incremento total fué de 17 puntos. El promedio para los niños de 5-6 años fué de 25.45 (d.e.=6.09), y los niños de 12 obtuvieron 47.38 (d.e.=3.9). El incremento total fué de 22 puntos. Ver fig. 1.

\begin{tabular}{c|ccccc}
\hline & & $5-6$ & $7-8$ & $9-10$ & $11-12$ \\
\hline \multirow{2}{*}{ Niños } & Media & 25.5 & 34.1 & 39.0 & 47.4 \\
& D.S. & 6.1 & 6.7 & 4.5 & 3.9 \\
\hline
\end{tabular}




\begin{tabular}{c|ccccc}
\hline Niñas & Media & 25.1 & 30.5 & 40.1 & 42.9 \\
& D.S. & 6.4 & 6.6 & 7.2 & 4.2 \\
Total & Media & 25.3 & 32.3 & 39.5 & 45.2 \\
& D.S. & 6.2 & 6.8 & 5.9 & 4.6 \\
\hline
\end{tabular}

Figura 1: Promedios por edad en la prueba de Boston.

En la EMVS no se encontraron diferencias entre sexos, pero si entre grupos de edad $\mathrm{F}(3,172)=10.67$, $\mathrm{p}<0.000$. En promedio las niñas de 5-6 años obtuvieron un puntaje de 6.45 (d.e.=1.47), en tanto que las niñas de 11-12 años alcanzaron 9.13 (d.e.=1.51). La diferencia entre los promedios de los grupos de edad de los niños fue ligeramente menor: los de 5-6 años tuvieron 6.5 (d.e.=1.97) y los de 11-12 lograron 9.13 (d.e.=1.51). Ver fig.2.

\begin{tabular}{c|ccccc}
\hline & & $5-6$ & $7-8$ & $9-10$ & $11-12$ \\
\hline \multirow{3}{*}{ Niños } & Media & 6.5 & 6.76 & 8.0 & 9.13 \\
& D.S. & 1.9 & 2.39 & 7.98 & 1.51 \\
& Media & 6.5 & 2.0 & 7.15 & 8.3 \\
& D.S. & 1.47 & 6.88 & 2.03 & 2.29 \\
& Media & 6.5 & 2.35 & 7.6 & 8.72 \\
& D.S. & 1.72 & 2.03 & 1.95 \\
\hline
\end{tabular}

Figura 2: Promedios por edad en la escala de memoria verbal secuencial.

Las diferencias entre los sexos en la PF no fueron significativas. Las diferencias entre los grupos de edad tuvieran significancia estadística $F(3,172)=32.48$, $\mathrm{p}<0.000$. Los niños de 5-6 años empezaron con pro- medios más bajos que las niñas de la misma edad (27.02 y 30.4 respectivamente), en tanto que los niños y las niñas de 11-12 años tuvieron promedios relativamente iguales (34.27 y 33.89). Ver fig. 3.

\begin{tabular}{c|ccccc}
\hline & & $5-6$ & $7-8$ & $9-10$ & $11-12$ \\
\hline \multirow{3}{*}{ Niños } & Media & 27.02 & 32.43 & 32.70 & 34.27 \\
& D.S. & 5.24 & 1.87 & 1.95 & 1.07 \\
& Media & 30.45 & 31.19 & 33.50 & 33.89 \\
& D.S. & 2.82 & 2.50 & 1.52 & 1.15 \\
& Media & 28.74 & 31.81 & 33.07 & 34.09 \\
& D.S. & 4.51 & 2.27 & 1.79 & 1.11 \\
\hline
\end{tabular}

Figura 3: Promedios por edad en la prueba de fichas.

En la FAS se realizó una revisión ortográfica (FASO) de la ejecución fonológica con el fin de evaluar el manejo de las reglas ortográficas. En ninguna de las versiones (FASF, FASO, FASS) se encontraron diferencias significativas entre los sexos. Por otra parte, se encontraron diferencias significativas en las tres versiones de la FAS entre los grupos de edad: FASF $\mathrm{F}(3,172)=54.5$, $\mathrm{p}<0.000$; FASO $\mathrm{O}(3,172)=59.4$, $\mathrm{p}<0.000$; FASS $\mathrm{F}(3,172)=63.0, \mathrm{p}<0.000$. La generación de palabras, en el grupo de 5-6 años, fue muy baja en las versiones fonológica y ortográfica de la prueba (niños: 2.46 , niñas: 2.15 y niños: 1.9 , niñas: 2.23 respectivamente). En el grupo de 11-12 años, en las versiones fonológica y ortográfica, los puntajes promedio fueron: niños: 8.17 , niñas: 7.51 y niños: 7.83, niñas: 7.3, respectivamente. La versión semántica de la prueba mostró promedios más altos en los niños y niñas tanto en el grupo de cinco años (niños: 6.86, niñas: 7.6) como en el de doce (niños: 14.52, niñas: 12.2). Ver figs. 4, 5, y 6.

\begin{tabular}{c|ccccc}
\hline & & $5-6$ & $7-8$ & $9-10$ & $11-12$ \\
\hline \multirow{3}{*}{ Niños } & Media & 2.15 & 4.92 & 5.44 & 8.17 \\
& Diñas & 2.12 & 1.67 & 2.36 & 1.95 \\
& Media & 2.46 & 4.21 & 5.68 & 7.51 \\
\multirow{3}{*}{ Total } & D.S. & 2.39 & 1.56 & 2.23 & 2.34 \\
& Media & 2.31 & 4.56 & 5.55 & 7.84 \\
& D.S. & 2.24 & 1.64 & 2.27 & 2.15 \\
\hline
\end{tabular}

Figura 4: Promedios por edad en la prueba fas fonológica.

\begin{tabular}{c|ccccc}
\hline & & $5-6$ & $7-8$ & $9-10$ & $11-12$ \\
\hline Niños & Media & 1.90 & 4.56 & 5.02 & 7.83 \\
\hline
\end{tabular}




\begin{tabular}{c|ccccc}
\multirow{4}{*}{ Niñas } & D.S. & 1.93 & 1.62 & 2.35 & 1.83 \\
& Media & 2.23 & 3.95 & 5.23 & 1.98 \\
\multirow{3}{*}{ Total } & D.S. & 2.32 & 1.46 & 5.12 & 2.27 \\
& Media & 2.06 & 4.25 & 2.17 & 7.57 \\
\hline
\end{tabular}

Figura 5: Promedios por edad en la prueba fas ortográfica.

\begin{tabular}{c|ccccc}
\hline & & $5-6$ & $7-8$ & $9-10$ & $11-12$ \\
\hline \multirow{3}{*}{ Niños } & Media & 6.86 & 9.38 & 11.11 & 14.52 \\
& D.S. & 1.82 & 2.42 & 2.34 & 2.19 \\
& Media & 7.60 & 8.98 & 11.85 & 12.20 \\
\multirow{3}{*}{ Total } & D.S. & 2.27 & 1.74 & 2.55 & 1.92 \\
& Media & 7.23 & 9.18 & 11.45 & 13.36 \\
& D.S. & 2.07 & 2.09 & 2.44 & 2.35 \\
\hline
\end{tabular}

Figura 6: Promedios por edad en la prueba fas semántica.

El análisis del manejo de las reglas ortográficas en los niños mostró diferencias pequeñas en el número de palabras reportadas en la modalidad fonológica y en la modalidad ortográfica. Las diferencias en porcentajes entre estas dos modalidades estuvo entre el $2.8 \%$ y el $11.7 \%$.
El análisis de varianza en FS demostró diferencias significativas entre los grupos de edad $\mathrm{F}(3,172)=$ $39.9, \mathrm{p}<0.000$, pero no entre niños y niñas. Los promedios de los niños y niñas de cinco años fueron casi iguales (11.8 y 11.36 respectivamente), en tanto que los promedios para los niños y niñas de 11-12 años fue de 14.0. Ver fig. 7.

\begin{tabular}{c|ccccc}
\hline & & $5-6$ & $7-8$ & $9-10$ & $11-12$ \\
\hline \multirow{3}{*}{ Niños } & Media & 11.86 & 13.24 & 13.83 & 14.0 \\
& D.S. & 1.98 & 1.30 & .39 & .00 \\
& Media & 11.36 & 13.48 & 13.80 & 14.0 \\
& D.S. & 1.97 & .93 & .52 & .00 \\
& Media & 11.61 & 13.36 & 13.81 & 14.0 \\
& D.S. & 1.97 & 1.12 & .45 & .00 \\
\hline
\end{tabular}

Figura 7: Promedios por edad en la prueba de figuras superpuestas.

En la figura 8 se presentan los resultados del análisis de varianza para cada una de las pruebas aplicadas. Se presentan los resultados por grupos de edad y por sexos. En todas las pruebas aplicadas se encontraron diferencias significativas en los grupos de edad. Con respecto a los sexos no hubo diferencias entre los promedios de ejecución de los niños y niñas en ninguna de las pruebas aplicadas.

\begin{tabular}{c|rrrrr}
\hline & Suma de cuadrados & $G l$ & Cuadrados mínimos & $F$ & $P$ \\
\hline Boston & & & & & \\
Edad & 10132.1 & 3 & 3377.3 & 97.7 & .000 \\
Sexo & 192.5 & 1 & 192.5 & 2.1 & n.s. \\
PMVS & & & & & \\
Edad & 130.5 & 3 & 43.5 & 10.6 & .000 \\
Sexo & 7.4 & 1 & 7.4 & 1.5 & n.s. \\
Fichas & & & & & \\
Edad & 722.7 & 3 & 240.9 & 32.4 & .000 \\
Sexo & 15.5 & 1 & 15.5 & 1.3 & n.s. \\
FASF & & & & & \\
Edad & 718.0 & 3 & 239.3 & 54.5 & .000 \\
Sexo & 2.9 & 1 & 2.9 & 0.3 & n.s. \\
FASO & & & & & \\
Edad & 706.3 & 3 & 235.4 & 59.4 & .000 \\
Sexo & 1.6 & 1 & 1.6 & 0.2 & n.s. \\
FASS & & & & & \\
Edad & 953.5 & 3 & 317.8 & 63.0 & .000 \\
Sexo & 6.2 & 1 & 6.2 & 0.6 & n.s. \\
FIG SUP & 158.0 & 3 & 52.6 & 39.9 & .000 \\
Edad & & & & & \\
\hline
\end{tabular}




\begin{tabular}{l|lllll} 
Sexo & 0.47 & 1 & 0.47 & 0.2 & n.s. \\
\hline
\end{tabular}

Figura 8: Resultados del análisis de varianza para todas las pruebas aplicadas.

Se calcularon los percentiles para cada una de las pruebas aplicadas. Las tablas de percentiles consideran únicamente los grupos de edad y no se presentan percentiles por sexos, ya que no se encontraron diferencias significativas entre sexos, sino solamente entre los grupos de edad.

\section{DISCUSIÓN}

Se encontraron diferencias únicamente entre los grupos de edad, pero no así entre el género.

Estos resultados apoyan el papel del desarrollo en la emergencia de las actividades cognoscitivas. El papel que juega el sexo en la aparición y desarrollo de los procesos psicológicos superiores parece ser nula. Estos datos concuerdan con los reportados en otras partes.

Sin embargo si consideramos que la muestra que participó en este estudio está conformada por niños de un colegio bilingüe, se tendría que considerar que el nivel de estimulación es más alto que el de un niño o niña que estudie en un colegio monolingüe. Por lo tanto las posibles diferencias esperadas entre género puede ser que no se manifiesten como ya se ha reportado en otros estudios (Kelly y Britton, 1996; Brito, Alfradique, Pereira, Porto y Santos, 1998). También se puede esperar que las diferencias entre niños con diferentes tipos de educación (bilingüe vs. monolingüe) sean más pronunciadas (Lopera, 1996).

Otro punto importante de revisar es la gran diferencia entre los promedios de los grupos de niños evaluados en este estudio y los promedios obtenidos por Ardila y Rosselli (1994), ya que ellos reportaron, en general promedios más altos en su estudio.

Una diferencia muy notoria es la encontrada en los puntajes de la Prueba de Fichas. Los niños de nuestro estudio obtienen en promedio 4 puntos porcentuales menos que los del trabajo realizado en Colombia.

Otro puntaje muy diferente es el observado en otra prueba de lenguaje: la de Vocabulario de Boston. De nueva cuenta los niños del presente estudio obtienen puntajes en promedio más bajos que los de los niños colombianos.

La explicación de estas diferencias en los resultados pueden ser:

a) Que los niños colombianos estén más orientados y/o estimulados verbalmente.

b) Que las características de las dos muestras sean realmente diferente debido a factores de tipo escolar. Los niños reportados por Ardila y Rosselli son monolingües y de dos niveles socioeconómicos diferentes (alto y bajo), en tanto que la muestra reportada en el presente trabajo estudia en un colegio bilingüe y son del nivel socioeconómico medio.

Sin embargo dado que este estudio es parte de un trabajo mucho más amplio es importante señalar que se continuará con la evaluación de niñas y niños de los mismos grupos de edad, pero con características socioeconómicas y con tipos de educación diferentes. Estas evaluaciones posteriores serán un punto de comparación para evaluar si el factor sociocultural o si el tipo de educación (monolingüe o bilingüe) juegan un papel determinante en la ejecución de estas pruebas neuropsicológicas.

\section{REFERENCIAS}

Ardila, A. \& Ostrosky-Solis F. (1991). Diagnóstico del daño cerebral. Un enfoque neuropsicológico. México: Trillas, 1991.

Ardila, A. \& Rosselli, M. (1988). Effects of educational level on linguistic abilities. Em Congreso de la National Academy of Neuropsychologists, Orlando.

Ardila, A. \& Rosselli, M. (1994). Development of language, memory, and visuospatial abilities in 5 to 12 year-old children using a neuropsychological battery. Developmental Neuropsychology, 10(2), 97-120.

Brito, G., Alfradique, G., Pereira, C., Porto, C. y Santos, T. (1998). Developmental norms for eigth instruments used in the neuropsychological assessment of children: studies in Brazil. Braz. J Med Biol Res. 31(3) 399-412.

Canseco, E. (1998). El esquema del diagnóstico neuropsicológico Ardila-Ostrosky-Canseco. Em E. Matute (Org.), Neuropsicología y educación especial (pp. 45-55). México: Gobierno de Jalisco.

Curro, G. (1992). Evaluación de la memoria tactil, visual y auditiva en niós de 7 a 12 años. Em I Encuentro Nacional de Neuropsicología, Puebla, México.

De Renz, E. \& Faglioni, P. (1978). Normative data and screening power of a shortened version of the Token Test. Cortex, 14, 327-342.

Ferrer, A. (1992). Evaluación neuropsicológica del desarrollo infantil. Perspectiva neuropsicológica. Em M. Rosselli \& A. Ardila (Org.), Neuropsicología infantil. Avances en investigación, teoría y práctica (pp. 125-15). Medellin: Prensa Creativa.

Galindo, G., Corte, J., Salvador, J., Ríos, B., Chatelain, L. \& SanEsteban, J. (1992). Fase piloto hacia la estandarización de la Figura Compleja de Rey-Osterrieth. Salud Mental, 4, 21-27.

Goodglass, H. \& Kaplan, H. (1986). Evaluación de las afasias y de trastornos similares. Buenos Aires: Editorial Médica Panamericana.

Nelly, T. Y Britton, P. (1996). Sex differences on an adaptation of the Digit Symbol subtest of the Wechsler Intelligence Scale for Children-III. Perceptual and Motor Skills, 83(3) 843-847.

Lezak, M.(1983). Neuropsychological assessment. New York: Oxford University Press.

Lopera, F. (1992). Evaluación neuropsicológica infantil. Perspectiva neurológica. Em M. Rosselli \& A. Ardila (Orgs.), Neurop- 
sicología infantil. Avances en investigación, teoría y práctica (pp. 97-123). Medellin: Prensa Creativa.

Luria, A. (1973). El cerebro en acción. Barcelona: Fontanella.

Ostrosky, F., Ardila, A., Canseco, E., Navarro, M. E., Quintanar, L. \& Meneses S. (1984). Evaluación neuropsicológica y factores socioculturales. Em XXIII Congreso Internacional de Psicología, Acapulco, México.

Rosselli, M., Ardila, A. \& Rosas P. (1990). Neuropsychological assessment in illiterates: II Language and praxic habilitéis. Brain and Cognition, 1, 281-296.

Spreen, O. \& Straus, E. (1991). A compendium of neuropsychological tests. Administration, norms and commentary. New York: Oxford University Press.
Valdez, P., Ramirez, C. \& Nava, G. (1992). Prueba de Figuras y Palabras. Una propuesta para la evaluación de la memoria. Em I Encuentro Nacional de Neuropsicología, Puebla, México.

Wechsler, D. (1945). A standarized memory scale for clinical use. Journal of Psychology, 19, 87-95.

Enviado: 02/06/2005 Revisado: 20/06/2005 Aceito10/07/2005

\section{Sobre os autores:}

M.A. Francisco Javier Galarza del Ángel: Laboratorio de Psicofisiología. Facultad de Ciencias Humanas. Universidad Autónoma de Baja California.

Mtro. Luis Alfredo Padilla López: Laboratorio para la Investigación del Comportamiento. Facultad de Ciencias Humanas. Universidad Autónoma de Baja California.

Mtra. Julieta Bonilla: Laboratorio de Psicofisiología. Facultad de Ciencias Humanas. Universidad Autónoma de Baja California.

Universidad Autónoma de Baja California Facultad de Ciencias Humanas - Blvds. Castellón y L. Toledano s/n Colonia Esperanza Agrícola C.P. 21350 - Mexicali, B.C. México. 
